\title{
Dosimetric evaluation of irregular surface compensator and intensity-modulated radiotherapy in breast radiotherapy
}

\author{
Gokcen Inan, Osman Vefa Gul, Hamit Basaran \\ Department of Keykubat Campus, Faculty of Radiation Oncology, Selouk University, Konya, Turkey
}

\begin{abstract}
Background: This dosimetric study aims to evaluate the dosimetric advantage of the irregular surface compensator (ISC) compared with the intensity-modulated radiotherapy (IMRT).

Materials and methods: Ten patients with whole breast irradiation were planned with the ISC and IMRT techniques. Six different beam directions were selected for IMRT and ISC plans. The treatment plans were evaluated with respect to planning target coverage, dose homogeneity index (DHI) and organs at risk (OARs) sparing. Monitor units (MUs) and the delivery time were analysed for treatment efficiency.

Results: The ISC technique provides a better coverage of the PTV and statistically significantly better homogeneity of the dose distribution. For the ipsilateral lung and heart, ISC and IMRT techniques deliver almost the same dose in all plans. However, MU counts and delivery time were significantly lower with the IMRT technique $(p<0.05)$.

Conclusion: For breast radiotherapy, when the ISC method was compared to the IMRT method, ISC provided better dose distribution for the target.
\end{abstract}

Key words: irregular surface compensator; intensity-modulated radiotherapy; breast radiotherapy

Rep Pract Oncol Radiother 2021;26(6):984-989

\section{Introduction}

Breast cancer is the most frequent cancer in women in developed countries at the present time. In a radiation oncology clinic, breast cancer constitutes approximately $25 \%$ of all cases [1]. Breast cancer treatments consist of surgery, radiation therapy, chemotherapy, and hormone therapy [2, 3]. The purpose of radiation therapy is to enable the best dose conformation to the planning target volume (PTV), while sparing critical organs [4]. For breast cancer, the shape of the PTV is irregular and concave. The adjacent organs at risk (OARs), including the heart and ipsilateral lung, make planning difficult for patients. In breast radiotherapy, more advanced methods have been advised to improve the homogeneity of dose distributions, such as intensity-modulated radiotherapy (IMRT) $[5,6]$. For breast IMRT plans, one of the techniques for intended coverage of the "flash region" is used for optimization of the nominal PTV followed by expansion of the fluence outside the skin using a skin flash tool [7]. Irregular surface compensator (ISC) is an electronic compensation algorithm performed in the treatment planning system (TPS) (Varian Medical Systems). Several studies have investigated the advantage of electronic compensation algorithms to improve dose homogeneity in the PTV $[8,9]$. However, most of them date from the period of pencil beam convolution algorithms that predict

Address for correspondence: Gokcen Inan, Selçuk Üniversitesi Faculty Of Sport Sciences, Department of Keykubat Campus, 42075 Konya, Turkey; e-mail: gokceninan85@gmail.com

This article is available in open access under Creative Common Attribution-Non-Commercial-No Derivatives 4.0 International (CC BY-NC-ND 4.0) license, allowing to download articles and share them with others as long as they credit the authors and the publisher, but without permission to change them in any way or use them commercially 
more homogeneous dose distributions in heterogeneous regions. In breast radiotherapy, accurate skin dose assessment is very important to provide skin dose. In this dosimetric study, we aimed to compare ISC and IMRT techniques with respect to the doses received by OARs, including the heart, ipsilateral lung, skin dose, dose homogenity index (DHI), conformity index (CI) and monitor units (MU) counts required for the treatment.

\section{Materials and methods}

\section{Patient selection and positioning}

Computed tomography (CT) images for a group of 10 randomly selected anonymous breast patients who underwent radical mastectomy operation in our clinic and were enrolled for this dosimetric study. Since the PTV and OAR values obtained for each technique were similar, the sample size was limited to 10. Ethics committee approval was not required since this was not a clinical study performed on patients, but a dosimetric simulation study. Informed Consent was not required since the dosimetric simulation study was performed on anonymous patient data. Patients were immobilized in a supine position on a breast board with the left arm up. The planning CT scans were performed using a CT scanner with a $3 \mathrm{~mm}$ slice thickness. CT images were transferred to TPS.

\section{Target volumes and organs at risk}

Treatment plans included supraclavicular lymph nodes and whole breast. All clinical target volumes (CTVs) and OARs were contoured according to the Radiation Therapy Oncology Group (RTOG) contouring atlas by a single radiation oncologist using Varian version 15.1 TPS software. PTV was created by expanding the CTV $5 \mathrm{~mm}$ isotopically. Anteriorly, the PTV was performed for being $2 \mathrm{~mm}$ inside the skin. OARs including the heart, lung, spinal cord, esophagus, and right breast were delineated by experienced radiation therapy oncologists.

\section{Treatment planning}

For each patient, IMRT and ISC treatment plans were created on the TPS. The technique for ISC using the fluence editor was used. The fluence editor allows the planner to modify fluence distribution of a field in beams eye view (BEV). We used a $1-\mathrm{cm}$ thick tissue equivalent compensator to the surface of the chest walls. Both treatment plans used the same isocenter and tangential field. For the IMRT and ISC, plans were generated with 6 coplanar fields with gantry angles. The most optimum beam angles were selected on the basis of the position to PTV and critical organs. Optimization algorithms were used to create fluence maps dose distributions. Treatment plans were performed with $6 \mathrm{MV}$ photon beams. The analytical anisotropic algorithm (AAA) was used for dose calculation. The prescribed dose was $5000 \mathrm{cGy}$ in 25 fractions for the target volume.

\section{Plan analysis}

Dose volume histograms (DVHs) obtained from treatment plans were evaluated for target volumes and OARs. Maximum and mean dose $\left(\mathrm{D}_{\max }\right.$ and $\left.\mathrm{D}_{\text {mean }}\right), \mathrm{HI}, \mathrm{CI}, \mathrm{MU}$ and delivery time were compared for PTV. For OAR, the values of interest in this study included Dmax, Dmean, V5 Gy, V10 Gy and V20 Gy for the heart and ipsilateral lung were evaluated. DHI was defined according to the International Commission on Radiation Units and Measurements Report 83. Dose homogeneity in the PTV was compared by means of the DHI [10].

$$
H I=\frac{\left(D_{2 \%}-D_{98 \%}\right)}{D_{50 \%}}
$$

In this formula $\mathrm{D}_{98}$ is the maximum dose absorbed in those $2 \%$ of the PTV least irradiated, $\mathrm{D}_{2}$ is the minimum dose absorbed in those $2 \%$ of the PTV most irradiated. CI is defined by the ratio of reference isodose volume to target volume of PTV [10]. The $95 \%$ isodose volume was taken as reference volume of the PTV. In addition, the skin dose obtained from three point marker on the skin was measured and compared with the dose values predicted by the TPS.

\section{Statistical analysis}

For statistical analysis (SPSS Inc. Chicago, II., USA) paired samples $\mathrm{T}$ test was used. A $\mathrm{p}$ value of $<0.05$ was considered to be a statistically significant difference.

\section{Results}

Statistical dosimetric evaluation for PTV coverage, HI, CI, MU and delivery time for all plans are tabulated in Table 1 . The ISC method was shown to improve homogeneity of the dose distribution 




Figure 1. Isodose curves of one representative patient for the intensity-modulated radiotherapy (IMRT) and for the irregular surface compensator (ISC)

in the PTV. The average maximum and mean doses for PTV were $53.99 \pm 0.97$ and $54.57 \pm 0.45$, $49.56 \pm 0.36$ and $49.76 \pm 0.60$ for ISC and IMRT plans, respectively. Figure 1 shows the evaluation of the 3 DCRT plan with IMRT plan. Concerning the calculated dose HI followed by the standard deviation, the DHI values for were $0.12 \pm 0.03$ and $0.15 \pm 0.04$ for ISC and IMRT, respectively. The heterogeneity index was significantly lower for ISC plans $(\mathrm{p}=0.001)$. Mean CI for the two techniques were $0.42 \pm 0.14$ and $0.66 \pm 0.04$, respectively. Statistically significant results for $\mathrm{CI}$ were observed between all treatment plans $(\mathrm{p}=0.001)$. The mean MU for the ISC and IMRT techniques were $1452 \pm 206$ and $1187 \pm 122$, respectively. Significant differences were observed for MU counts between all treatment plans $(\mathrm{p}=0.001)$. IMRT plans deliver fewer MUs than ISC plans. The delivery time (in minutes) was as long for ISC plans as it was for IMRT plans $(\mathrm{p}=0.002)$. The statistical dosimetric evaluation for OARs is listed in Table 2. When compared to the OARs, there was no significant difference. Figure 2 shows dose-volume histogram comparison of the ISC with 3DCRT techniques. The heart volume receiving 10 to $20 \mathrm{~Gy}$ was lower in the ISC than in IMRT plans, but this was not statistically significant. When ISC plans were compared with IMRT plans in the TPS at the same point, the average dose of patient's breast region (medial and lateral tangential fields) were $50.81 \pm 0.78$ and $50.15 \pm 0.90$ for the ISC plans, $49.76 \pm 1.14$ and $49.03 \pm 0.90$ for the IMRT plans. Significant differences were observed for skin doses between the two treatment plans $(\mathrm{p}=0.006, \mathrm{p}=0.000)$.

\section{Discussion and conclusion}

The main aim of this study was to compare two techniques of whole breast radiotherapy with respect to target coverage and OAR doses. In this study the skin dose of patients was measured using point dose measurement options in the TPS. Individual beamlets with dynamic multileaf collimators were recommended a few years ago, between conventional fields and IMRT to develop dose distributions in whole breast [12]. Several studies have been performed on the impact of this method to improve dose homogeneity in comparison to other techniques $[13,9]$. While trying to plan to increase dose homogeneity in the target could lead to increased lung doses and hotspots that may result in higher skin problems. According to the results obtained from this study, the ISC technique led to improving PTV dose coverage and reducing dose heterogeneity. Also, the ISC technique reduced hotspots for breast radiotherapy. Increasing target coverage is very substantial for breast patients for whom cold spots in PTV should be avoided.

Flejmer et al. compared the clinical effects for the ISC technique with 3D-CRT for breast radiotherapy. 


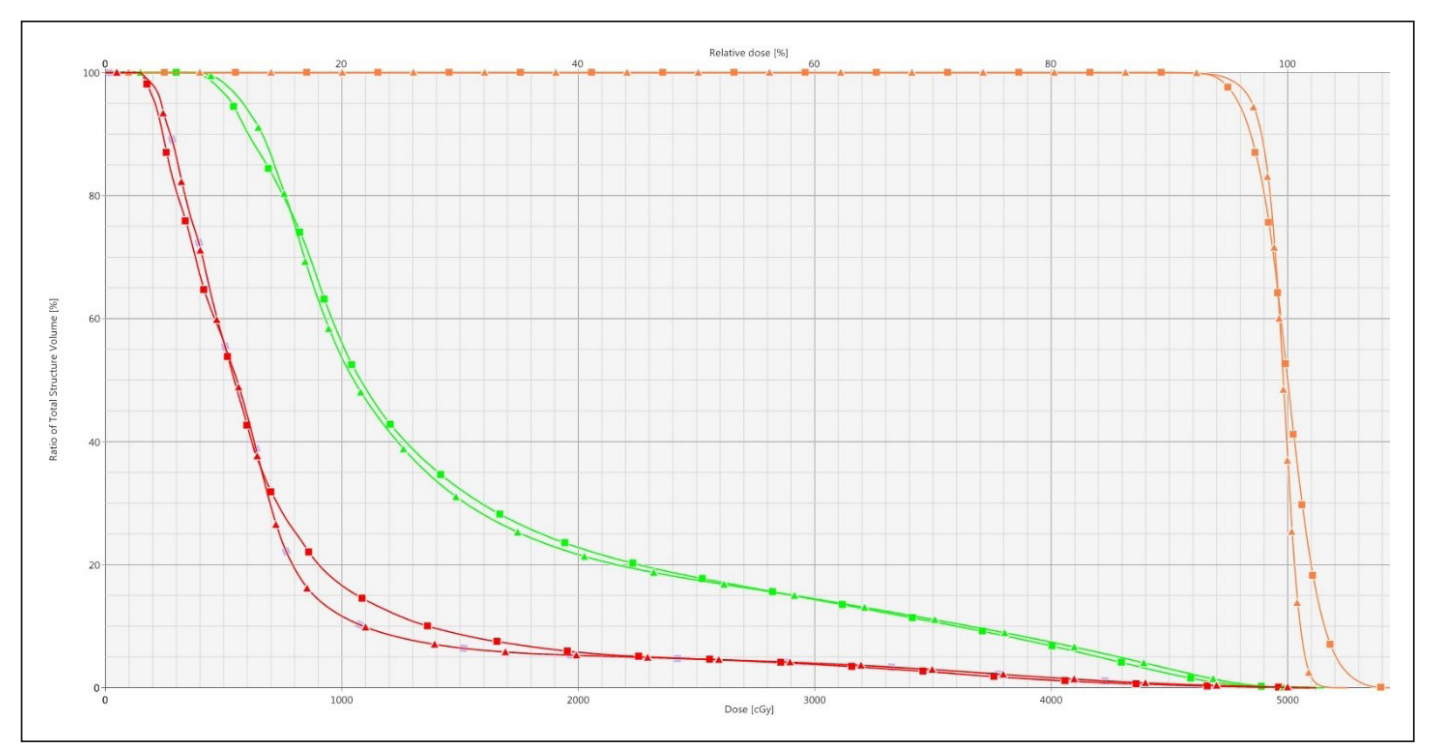

Figure 2. Dose-volume histogram comparison of the irregular surface compensator (ISC) with the intensity-modulated radiotherapy (IMRT) technique. Orange: planning target volume (PTV), green: ipsilateral lung, red: heart $\mathbf{\Delta}$ : ISC technique and घ: IMRT technique

They reported that when compared with ISC and 3DCRT plans, the ISC method produced statistically significantly better homogeneity of the dose distribution. And also, they found that the heart and contralateral lung received a similar dose [14].

Kuwahata et al. compared the dosimetric benefits of the ISC technique for whole breast radiotherapy with the field-in-field (FIF) techniques. They indicated that the ISC technique with a fluence editor allowed significant improvements in dose distribution compared with the FIF technique. They found that the ISC method significantly decreased cold spot regions while suppressing Dmax equal to or less than the FIF technique. Therefore, they concluded that the ISC technique is useful for whole breast radiotherapy [15].

Hideki et al. compared the dosimetric advantage of breast radiotherapy between ISC and a conventional tangential field method using physical wedges. They reported that the ISC method for breast radiotherapy provides apparently better dose distribution in the PTV [16].

Dyer et al. compared the dosimetric advantage of T1aN0M0, Stage IA vulva cancer to illustrate the progressive vulvar swelling and lymph edema seen during treatment between ISC and standard RT planning techniques [17]. They reported that standard planning techniques to treat vulvar cancer patients with IMRT do not sufficiently account for the change in patient anatomy and can lead to a marginal miss. They found that ISC was an RT planning technique that can decrease the risk of a marginal miss and the technique was easily implemented during the planning stages of RT treatment. They suggested that the use of an ISC technique can improve vulvar clinical target volume coverage and plan homogeneity.

Sung et al. evaluated the dosimetric advantages of the ISC technique on gynecologic cancer patients [18]. They found that the ISC technique has the superior target coverage and healthy tissue sparing in comparison with conventional four-field box and comparable normal organ saving compared to IMRT5F. They suggested that ISC can be an available option for gynecologic radiotherapy.

The current study has several limitations. This is a dosimetric study, and it does not include vital aspects required for clinical use. The number of patients used for comparison was limited to 10 , this may be improved in the next study to obtain a better sample. $4 \mathrm{D}$ technique for breast motions influence dose distribution; however, we evaluated the two methods without including the influence of them.

In our clinic, as a countermeasure for breast motion, all patients received breath coaching before CT scanning and treatment. As a countermeasure for breast motion, we took MV images for patient positioning before every fraction of the treatment and that was performed both anterior-posterior 
and lateral. Several studies have suggested that breast motions can affect the dose distribution with IMRT $[19,20]$. However, Furuya et al. evaluated the dosimetric impact of respiratory motion and setup error on whole breast tangential irradiation [21]. They found that the dosimetric impacts of breast motions and daily setup errors for ISC in the whole fraction are relatively small and, thus, clinically acceptable. Radiotherapy planners at many institutions are unfamiliar with the ISC technique. Based on the dosimetric results in this study, there is a clinical advantage for the ISC technique compared with IMRT for breast cancer. The ISC method enables a reduction in hot regions without increasing cold regions. ISC technique was found to be the influential to maintain in target coverage. ISC plans improve the conformity and homogeneity of the dose distribution in PTV. The ISC method for breast treatments provides significantly better dose distribution in the target volume and the ISC method should be considered as a useful alternative for breast treatment.

\section{Conflict of interests}

The authors have no conflicts of interest to declare.

\section{Funding}

The authors declared that this study has received no financial support.

\section{References}

1. Perez C, Brady WL, Halperin CE, Wazwe DE. Principles and Practice of Radiation Oncology. 7th ed. Wolters Kluwer : 3962-3963.

2. Liu MJ, Hawk H, Gershman ST, et al. The effects of a National Breast and Cervical Cancer Early Detection Program on social disparities in breast cancer diagnosis and treatment in Massachusetts. Cancer Causes Control. 2005; 16(1): 27-33, doi: 10.1007/s10552-004-1289-4, indexed in Pubmed: 15750855.

3. Katz SJ, Lantz PM, Janz NK, et al. Patterns and correlates of local therapy for women with ductal carcinoma-insitu. J Clin Oncol. 2005; 23(13): 3001-3007, doi: 10.1200/ JCO.2005.04.028, indexed in Pubmed: 15860856.

4. Low DA, Moran JM, Dempsey JF, et al. Dosimetry tools and techniques for IMRT. Med Phys. 2011; 38(3): 1313-1338, doi: 10.1118/1.3514120, indexed in Pubmed: 21520843.

5. Chui $C S$, Hong $L$, Hunt $M$, et al. A simplified intensity modulated radiation therapy technique for the breast. Med Phys. 2002; 29(4): 522-529, doi: 10.1118/1.1460875, indexed in Pubmed: 11991123.

6. James HV, Scrase CD, Poynter AJ. Practical experience with intensity-modulated radiotherapy. Br J Radiol. 2004;
77(913): 3-14, doi: 10.1259/bjr/14996943, indexed in Pubmed: 14988132.

7. Sankar A, Velmurugan J. Different intensity extension methods and their impact on entrance dose in breast radiotherapy: A study. J Med Phys. 2009; 34(4): 200-205, doi: 10.4103/0971-6203.56079, indexed in Pubmed: 20098549.

8. Caudell JJ, De Los Santos JF, Keene KS, et al. A dosimetric comparison of electronic compensation, conventional intensity modulated radiotherapy, and tomotherapy in patients with early-stage carcinoma of the left breast. Int J Radiat Oncol Biol Phys. 2007; 68(5): 1505-1511, doi: 10.1016/j.jijrobp.2007.04.026, indexed in Pubmed: 17674981.

9. Emmens DJ, James HV. Irregular surface compensation for radiotherapy of the breast: correlating depth of the compensation surface with breast size and resultant dose distribution. Br J Radiol. 2010; 83(986): 159-165, doi: 10.1259/ bjr/65264916, indexed in Pubmed: 19752168.

10. Yoon M, Park SY, Shin D, et al. A new homogeneity index based on statistical analysis of the dose-volume histogram. J Appl Clin Med Phys. 2007; 8(2): 9-17, doi: 10.1120/ jacmp.v8i2.2390, indexed in Pubmed: 17592460.

11. Paddick I. A simple scoring ratio to index the conformity of radiosurgical treatment plans. J Neurosurg. 2000; 93(Suppl_3): 219-222, doi: 10.3171/jns.2000.93.supplement_3.0219.

12. Chui CS, Hong L, Hunt M, et al. A simplified intensity modulated radiation therapy technique for the breast. Med Phys. 2002; 29(4): 522-529, doi: 10.1118/1.1460875, indexed in Pubmed: 11991123.

13. Caudell JJ, De Los Santos JF, Keene KS, et al. A dosimetric comparison of electronic compensation, conventional intensity modulated radiotherapy, and tomotherapy in patients with early-stage carcinoma of the left breast. Int J Radiat Oncol Biol Phys. 2007; 68(5): 1505-1511, doi: 10.1016/j.jijrobp.2007.04.026, indexed in Pubmed: 17674981.

14. Flejmer AM, Josefsson D, Nilsson $M$, et al. Clinical Implications of the ISC Technique for Breast Cancer Radiotherapy and Comparison with Clinical Recommendations. Anticancer Res. 2014; 34: 3563-3568, indexed in Pubmed: 24982370.

15. Kuwahata N, Fujita H, Yamanishi H, et al. Dosimetric Comparison of Irregular Surface Compensator and Fieldin-Field for Whole Breast Radiotherapy. J Med Phys. 2018; 43(2): 79-84, doi: 10.4103/jmp.JMP_73_17, indexed in Pubmed: 29962684.

16. Hideki F, Nao K, Hiroyuki $\mathrm{H}$, et al. Improvement of dose distribution with irregular surface compensator in whole breast radiotherapy. J Med Phys. 2013; 38(3): 115-119, doi: 10.4103/0971-6203.116361, indexed in Pubmed: 24049317.

17. Dyer BA, Jenshus A, Mayadev JS. Integrated skin flash planning technique for intensity-modulated radiation therapy for vulvar cancer prevents marginal misses and improves superficial dose coverage. Med Dosim. 2019; 44(1): 7-10, doi: 10.1016/j.meddos.2018.01.003, indexed in Pubmed: 29500108.

18. Sung W, Kim JI, Kim HS, et al. Performance of the irregular surface compensator compared with four-field box and intensity modulated radiation therapy for gynecologic 
cancer. Phys Med. 2016; 32(12): 1537-1542, doi: 10.1016/j. ejmp.2016.10.025, indexed in Pubmed: 27866896.

19. Xing L, Crooks S, Li JG, et al. Incorporating respiratory motion into the design of intensity maps in IMRT treatment of breast cancer. Int J Radiat Oncol Biol Phys. 2000; 48(3): 199, doi: 10.1016/s0360-3016(00)80192-3.

20. Cao J, Roeske JC, Chmura SJ, et al. Calculation and prediction of the effect of respiratory motion on whole breast radiation therapy dose distributions. Med Dosim. 2009;
34(2): 126-132, doi: 10.1016/j.meddos.2008.07.002, indexed in Pubmed: 19410141.

21. Furuya $T$, Sugimoto $S$, Kurokawa $C$, et al. The dosimetric impact of respiratory breast movement and daily setup error on tangential whole breast irradiation using conventional wedge, field-in-field and irregular surface compensator techniques. J Radiat Res. 2013; 54(1): 157-165, doi: 10.1093/jrr/rrs064, indexed in Pubmed: 22859565 . 